\title{
Pregnancy Termination: Experiences of Women Seeking Post-Abortion Care Services in Ghana
}

Kenneth Setorwu Adde ( $\square$ kensadde@gmail.com )

University of Cape Coast https://orcid.org/0000-0003-2475-7326

Eugene Kofuor Marfo Darteh

University of Cape Coast

Akwesi Kumi-Kyereme

University of Cape Coast

Research article

Keywords: Post-Abortion Care; Spontaneous Abortion; Induced Abortion Experiences; Pregnancy

Termination; Health-Seeking Behavior; Ghana

Posted Date: April 24th, 2020

DOI: https://doi.org/10.21203/rs.3.rs-24241/v1

License: (c) (1) This work is licensed under a Creative Commons Attribution 4.0 International License.

Read Full License 


\section{Abstract}

Background: Although abortion is a global health concern, it has a higher negative impact on developing countries due to controversial perspectives and varied regulatory regimes. Depending on whether it is induced or spontaneous, women encounter varying experiences. This study examined the pregnancy termination experiences of women seeking post-abortion care services.

Methods: An in-depth interview guide was used to collect data from 20 post-abortion care clients at the Volta Regional Hospital. Data was analyzed manually using a qualitative content analysis technique.

Result: The study found that medical abortion was main method of pregnancy termination used by women who had induced abortion. Spontaneous abortion, however, was attributed mainly to engaging in activities that required the use of excessive energy and travelling on bad roads by pregnant women. The study also revealed that women do not seek early post-abortion care services due to stigma and poverty.

Conclusion: To encourage women to seek early treatment, the Ministry of Health and the Ghana Health Service should take pragmatic steps in educating women on the dangers associated with delayed postabortion care services and the factors that expose women to spontaneous abortions.

\section{Background}

Globally, about 830 women die from pregnancy and childbirth-related complications every day, and abortion accounts for eight percent of these maternal mortality cases [1]. Abortion is one of the public health concerns globally, with its grave effects particularly in developing countries where there are controversial perspectives as well as varied regulatory regimes [2]. Although abortion rates in the developing world have declined significantly since the 1990s, they remain available on religious, moral and political beliefs rather than the medical needs of a woman [3].

In Ghana, abortion is one of the main contributors to the maternal mortality rate [4]. More than one in every ten maternal deaths is attributed to unsafe abortion [5]. The high rate of maternal mortality as a result of unsafe abortions can be attributed to social barriers such as provider attitudes[6], conscientious objection and the high stigma [7] attached to abortion in Ghana. These barriers make it difficult to effect appropriate policy reforms and changes that are necessary to address the problems posed by unsafe abortion [7]. Hence, women face many challenges in seeking abortion care [8]. It is, however, worth mentioning that abortion complications do not only result from unsafe abortions as both safe and unsafe abortions contribute to abortion complications [9]. Depending on the frequency, method, and location for seeking an abortion, women tend to have vast experiences motivated by diverse factors [9].

Studies have indicated that most induced abortions are results of unintended pregnancies and, maybe, mistimed pregnancies or possibly unwanted pregnancies; all these have their associated experiences (see Warriner \& Shah[10], and Finer et al., [11]). A woman who seeks a clandestine abortion may try some traditional techniques of varying efficacies and harmfulness, and these have their own experiences [12]. 
Some methods of unsafe abortion include drinking toxic fluids such as turpentine and bleach or other drinkable concoctions mixed with livestock manure such as cow dung [12]. Others are a forceful manipulation of the abdomen and insertion of sticks and other objects into the vagina, cervix or uterus [13]. Many of these techniques pose threats to a woman's health and, sometimes, even her life [13]. If these methods fail to cause a complete pregnancy termination, the woman may then go to a pharmacist, nurse or doctor known to provide abortion services [13].

According to the 2017 Ghana Maternal Health Survey, twenty percent of women aged 15-49 have ever induced an abortion. However, the percentage of women who have had an inducedabortion in the last 5 years preceding the 2007 and 2017 surveys remain relatively unchanged with five percent and seven percent respectively [14]. A study conducted in Southern Ghana reported 17 abortions per 1,000 women of reproductive age [15] while another found that about twenty-three percent of women have ever had an induced abortion [16].

Although abortion is a criminal offence in Ghana, it is permitted when the pregnancy is a result of rape or incest, when there is a need to protect the mental or physical health of the mother,and when there is a malformation of the fetus,thus, making the abortion law in Ghana relatively liberal compared to other African countries [7]. Nonetheless, more than two-thirds of women who sought abortion turned to untrained providers or induced abortion on their own $[17,18]$. Concerning common abortion practices, findings from other studies suggest medical abortion through the use of Mifepristone and Misoprostol in Ghana $[19,17,20]$. Data from the Ghana Maternal Health Survey [14] showed thirty-eight percent of the women who induced abortions indicated they used medical abortion, twenty-seven percent used nonmedical methods and twenty-four percent used dilation and curettage (D\&C) or dilation and evacuation (D\&E) while ten percent used other medical methods. Less common methods include inserting an object, herbs or other substances into the vagina; and drinking herbal concoctions $[17,20]$. The latter methods of abortion are associated with a number of complications thus requiring some post abortion care.

To help deal with abortion complications and its effects on maternal mortality and morbidity rates, a policy on post-abortion care was introduced in Ghana in 2003 [21]. In 2006, the Ghana Health Service and the Ministry of Health also published protocols for the provision of safe abortion services to help reduce the barriers to safe abortion. This was to help reduce abortion-related mortality and morbidity[22]. However, abortions remain one of the highest contributors to maternal deaths [4]. Though Ghana has made efforts to increase access to post-abortion care, the quality of service is still poor, underfunded and has low visibility [23]. It is, however, important to promote an equitable and reasonable health care seeking environment to improve reproductive and maternal health in Ghana.

Various issues concerning abortion have been studied in Ghana. For instance, studies have been done on the practice of abortion in Ghana(see Morhe et al., [24]; Sundaram, et al., [25]; GSS [20]) and attitudes towards abortion. Others have focused on post-abortion experiences in Ghana (see Esia-Donkoh et al. [26] and Rominski et al. [27]). Esia-Donkoh et al. [26] targeted women who received safe abortion services at a health facility while Rominskiet al.[27] focused on post-abortion contraception choices of women. This 
notwithstanding, there appears to be a paucity in the literature on the health-seeking behavior of women who sought post-abortion care. The current study, therefore, seeks to explore the pregnancy termination experiences of women who received post-abortion care services. This will enable us to have a clear view of the health-seeking behavior of women who have had unsafe and spontaneous abortions, and suggest feasible measures to improve post-abortion care services and maternal health care.

\section{Methods}

This study was part of a larger study that was intended to explore views of clients on post-abortion care in Volta Regional Hospital. Other issues covered in the study include abortion complications reported at the facility, post-abortion care services rendered to clients at the facility and client's satisfaction at the Volta Regional Hospital.

The study relied on the interpretivist paradigm on the backdrop that, this provides a better way of understanding the real-life experience of post-abortion care clients with respect to what they consider as their pregnancy termination experiences. The data collection was done at a Regional level Health facility in Ghana. A regional health facility in Ghana serves as a secondary referral point for all health facilities in a Region. The selected hospital is one of the state-owned modern Regional referral Hospitals. Obstetrics and gynecology care are some of the critical services available at the facility. The Ghana Maternal Health Survey of 2007 indicates that the Region has one of the highest incidences of induced abortions. About twelve percent of women in their reproductive age had ever had an abortion. Of this, a high percentage of about ninety-five indicate they could not get access to abortion services at health facilities.

Clients who were at the facility to seek post-abortion care services were purposefully selected with the help of the health professionals. These women were included in the study to help ascertain their experiences with abortion. Women who were willing to partake in the study were provided with consent forms to sign/thumbprint. They were then allowed to select their place and time of convenience for the interview to be carried out. The interviews were recorded and transcribed. On the average, an interview lasted for about 45 minutes. A sample size of 20 women made up of 8 clients who induced abortions and 12 clients who had spontaneous abortions was achieved through the principle of saturation. Guest, Bunce, and Johnson (28) theorize that a sample size of six interviews could be enough for the development of meaningful themes and useful interpretations. This study, therefore, deemed responses to be saturated when no new themes were emerging.

The interview was conducted with an in-depth interview guide. The guide had two sections ( $A$ and $B$ ). Section $A$ focused on the background characteristics of the participants and section $B$ focused on the experiences with pregnancy termination and the support received from family and friends. Respondents were asked to describe their pregnancy termination experiences. These formed the abortion strategies and the spontaneous abortion experiences of the respondents. Respondents were probed further on what informed their decisions to seek post-abortion care and if they received any form of support from 
anybody while seeking post-abortion care. The interview guide was developed by the authors after reviewing literature related to the topic under study.

Ethical clearance for the study was obtained from the Ghana Health Service Ethical Review Committee (GHS-ERC: 14/10/15). Permission to conduct the study was also obtained from the management of the hospital. Clients who agreed to take part in the study were required to sign/thumbprint an informed consent form before the interview was conducted.

\section{Analysis}

Data were analyzed manually using a qualitative content analysis technique. A qualitatively oriented text analysis based on the principles of the hermeneutic circle was adopted. To ensure the findings of the study were credible and unbiased, interview data were transcribed by an experienced researcher and compared to the tape interviews by a member of the research team. The data was coded independently by an expert qualitative data coder and the lead author. To ensure intercoder concordance, the authors and the expert qualitative data coder met afterward and reviewed the coding outcomes. Two experts in qualitative research crosschecked the codes identified for consistency. Following the jointly reviewed codes, themes and emerging issues were developed to mirror the narratives and interpret the data. Finally, quotes from the participants were used to corroborate the issues discussed.

\section{Results}

\section{Socio-demographic characteristics of participants}

Table 1 presents the socio-demographic characteristics of the participants. Sixteen respondents were in their $20 \mathrm{~s}$ while three were aged 30-39 years. Those who had attained basic education were seven whereas six had tertiary education. Nineteen of the participants were Christians while one was a Muslim. Eleven were never married while nine were married at the time of the study. The participants were maily dressmaker (5), civil servant (5), and traders (4). 
Table 1

Socio-demographic characteristics of participants.

\begin{tabular}{|c|c|c|}
\hline Variable & Frequency $(n=20)$ & Percentage (\%) \\
\hline \multicolumn{3}{|l|}{ Age } \\
\hline$<20$ & 1 & 5.0 \\
\hline $20-29$ & 16 & 80.0 \\
\hline $30-39$ & 3 & 15.0 \\
\hline \multicolumn{3}{|c|}{ Level of education } \\
\hline No education & 2 & 10.0 \\
\hline Primary & 3 & 15.0 \\
\hline JHS & 7 & 35.0 \\
\hline SHS & 2 & 10.0 \\
\hline Tertiary & 6 & 30.0 \\
\hline \multicolumn{3}{|l|}{ Religion } \\
\hline Christian & 19 & 95.0 \\
\hline Muslim & 1 & 5.0 \\
\hline \multicolumn{3}{|l|}{ Marital status } \\
\hline Never married & 11 & 55.0 \\
\hline Married & 9 & 45.0 \\
\hline \multicolumn{3}{|l|}{ Occupation } \\
\hline Dressmaker & 5 & 25.0 \\
\hline Trader & 4 & 20.0 \\
\hline Farmer & 2 & 10.0 \\
\hline Civil servant & 5 & 25.0 \\
\hline Student & 2 & 10.0 \\
\hline Hairdressing & 2 & 10.0 \\
\hline Total & 20 & 100.0 \\
\hline
\end{tabular}

\section{Abortion Experiences Of Respondents}


Abortion experiences among women include the range of decisions they had to make, their emotions, physical experiences, strategies they used including health care advice and dealing with clandestine medical abortions[25]. The study revealed a wide range of experiences including abortion strategies, spontaneous abortion experiences, decision making processes to seek care for abortion complications, and support received from family and friends during the abortion processes.

\section{Abortion Strategies}

Medical abortion - Mifepristone and Misoprostol is widely used by women in an attempt to terminate pregnancy [19]. The results revealed that the respondents usually induced abortion at home using the medical abortion method which involved swallowing of pills. According to some of the respondents, they had access to pills from other people, especially friends. A client who had used a pill to terminate a pregnancy shared her experience as follows:

"I took some medicine. I was discussing the pregnancy with a friend and she told me she knows some medicine, so she will bring it to me. She brought me the medicine and I used it." -Seamstress, 2029 years.

Adding to this, some of the respondents themselves were well aware of the pills they had to take to terminate the pregnancy. A client who got the pills herself had this to say:

"I just bought some medicine and took it. The medicine is called Cytotec. After taking it, I stayed indoors for a while I think for about 8 hours and I started feeling cramps and not long after that the blood started flowing." - Caterer, 20-29 years.

These experiences indicate that some women who induce abortion resort to medical abortion and may seek information regarding the pills to be taken from friends. The findings show that women usually prefer medical abortions at home as compared to seeking a medical abortion at health facilities. This could be a result of women's attempts to avoid the stigma attached to abortions.

\section{Spontaneous Abortion Experiences}

The study found that not all women deliberately induce abortion. The data revealed that twelve out of the twenty respondents had their pregnancies terminated unintentionally. Among the respondents who had their pregnancy terminations classified as spontaneous abortions, two main sources were observed. First, those whose pregnancies were aborted as a result of a second party second, those who lost their pregnancies unintentionally through their actions. The following extracts demonstrate the experiences of respondents who experienced spontaneous abortions as a result of a second parties.

"We were given a dewormer at... after we took the medicine ... our headmaster told us that the medicine aborts pregnancy." -Student, $<20$ years. 
Another closely related view to the above was the view of one woman who also indicated that her abortion was a result of physical abuse by her husband. She had this to say: "For this abortion, I will say it is my husband that gave me problems that resulted in the abortion... We were struggling with each other and someone else came to hit me from behind on my waist. It was his girlfriend who did that." Seamstress, 20-29 years.

Some other respondents had their pregnancies terminated by their actions though unintentional. Some of the respondents expressed experiencing spontaneous abortions as a result of their engagement in activities that demand the use of excessive energy such as cooking some meals and farming activities. A respondent had this to say:

"Hmmm, I don't know how to say it. I went to the farm to do some weeding. I was back from the farm and I realized I was bleeding so I came to the hospital and as soon as I got here the fetus itself came out." Student, 20-29 years.

Another respondent indicated that she noticed there was something wrong with her pregnancy while preparing a local meal called "banku". Although the preparation of this meal requires the use of excessive energy, it is likely the abortion resulted from other health issues. The respondent had this to say:

“... I was preparing 'banku' [a local meal] when the blood poured out of me. I couldn't even continue cooking the 'banku'. I had to leave it on the fire..." - Hairdresser, 20-29 years.

Some other respondents also experienced spontaneous abortions as a result of traveling on bad roads. To such respondents, their pregnancies got terminated because of the bumpy nature of the roads in their communities. The major means of traveling used by these respondents was the motorbike. One respondent surmised:

“... I can't exactly tell what happened but I did some rounds traveling around to my dad's place and other places on some very bad roads with a motorbike so when I came back, I was at health insurance [office] when I started bleeding..." -Fashion designer, 20-29 years.

To buttress this quotation, another respondent had this to say: "... I took a ride on a motorbike and it bumped into a pothole and I started feeling pains in my stomach only to come to the hospital and be told I have lost the pregnancy." - Trader, 20-29 years.

From the above, it is evident that the women associate the loss of their pregnancies to the activities they were engaged in prior to the pregnancy loss. These various activities demand the use of excessive energy or traveling on bad roads. Although these activities could be contributory factors to the pregnancies lost, we also believe there might be some other medical conditions that could have been aggravated by these activities to result in pregnancy losses.

One other respondent indicated that her spontaneous abortion was as a result of an accident that occurred at home. She fell, and this caused some pregnancy complications which later resulted in the 
unintentional termination of the pregnancy. She said:

"I slipped on the floor. Where we were staying at first had a cemented floor so when it rained the floor was slippery and I slipped and fell. I started bleeding so I went to the hospital." -Fashion designer, 2029 years.

\section{Factors that Influence the Decision to Seek Post-Abortion Care}

The study observed that different situations informed women's decisions to seek PAC services. Some of the women decided to seek PAC only at the point where they felt the pain were becoming unbearable and needed professional help in dealing with their situations. A respondent had this to say:

"My stomach was aching severely and I went to the hospital. It started as some normal pains but was increasing so when I couldn't bear it any longer, I went to the hospital." -Student, < 20 years.

One other respondent who also waited till the pain became severe and unbearable before seeking PAC services had this to say: "Well! When the thing [abortion] happened, I didn't come to the hospital immediately. I stayed home for some time and I started feeling pains in my waist. I went to urinate and saw blood in my underwear. When the pain was becoming too much, I pushed. After pushing, the blood started coming out of me like water. I almost died out of that. The blood was just coming out like water "tsruuuuuuuuuu" for a long time so I became weak and when I try to get up, I will fall." Seamstress/trader, 20-29 years.

Although the women were in pain, they were reluctant to seek early treatment from a health facilities and delayed until the pain was unbearable. This could be attributed to the fact that women feared being stigmatized for having experienced abortions. It could also be that these women were not aware of their right to comprehensive abortion care and as such were scared to seek early post-abortion care.

Women also delayed in seeking post-abortion care services at the hospital for various reasons. One of these reasons is the lack of or expiration of their National Health Insurance Scheme cards. This prevented some women from seeking care at the hospital since they did not have money to pay for the services at the health center. One woman had this to say: "... if not for the fact that my NHIS card had expired, I would have come earlier and not wait for it to become this serious." - Hairdresser, 20-29 years.

The study also observed that women who experienced complications from abortions were scared of stigma by society. This, to a large extent, affects the decision making of some women to seek help at healthcare facilities. Their position was that they would not want anyone to know they have had an abortion; hence, they prefer to hide their situation and suffer in silence. A respondent noted:

"The thing became unbearable for me before I decided to go to the hospital. I was feeling pains but I was feeling shy and didn't want anybody to know what was happening to me so I refused to go to the 
hospital.But later on, I couldn't breathe again so they had to charter a taxi and take me to the hospital." Seamstress, 20-29 years.

Nonetheless, multigravida women did not take long in seeking care at the hospitals. They were quick to report at the hospitals when they noticed a change or felt something was not in the right order. A multigravida respondent noted:

"I know that during pregnancy you are not supposed to see blood. I have given birth before and I know the process so immediately I saw something come out of me I knew something was wrong and I had to go to the hospital." - Fashion designer, 20-29 years.

To corroborate this, another multigravida respondent noted: "I was feeling cold and all of a sudden, I started feeling dizzy. I realized something was wrong with me so I was rushed to the hospital." -Farmer, 30-39 years.

The findings show that various factors such as lack of or the expiration of National Health Insurance and the fear of stigma are factors that delay the health-seeking behavior of women seeking post-abortion care. However, multigravida women were quick to report for early post-abortion care. Seeking early postabortion care by the multigravida women could be as a result of earlier experiences with pregnancies. The multigravida women could have been opportune to learning the importance of reporting for early treatment during antenatal care for their previous pregnancies.

\section{Support Received from Family and Friends}

We observed that sixteen of the women received support from their partners, family, and parents in seeking post-abortion care at the hospital. This support mainly came in the form of financial and emotional support. In most cases, those who received support from their partners and families acknowledged that the support was very critical in their decisions to seek care as well as their general experiences with the procedure. For instance, a respondent had this to say:

"Yes, my husband. He has been very helpful right from the beginning till now. He was there for me by going to the hospital with me and when I was admitted, he came to visit regularly and bought food for me as well as being supportive in comforting and consoling me to go through the process." -Caterer, 2029 years.

To corroborate the quote above, another respondent who received financial support for PAC from her family and partner had this to say: "Yes my father was a watchman and he is now on pension but takes some monthly pay of about GH\$800 so he went for it and the boy who impregnated me also added some as well as my elder brother and they took me to the hospital. They also visited me from time to time." Student, $<20$ years.

On the other hand, four of the respondents did not have any support from their immediate families and partners for PAC services. This resulted in being left stranded at the hospitals since they could not afford 
their bills. Consequently, they were kept at the hospitals against their will although they had fully recovered from their conditions. This was what a respondent had to say:

"No one helped me.Even as I am here right now, I have been discharged but because of my bills, I can't go home. Mysister was the one who got me a taxi but even that there is a man in our house who is a driver so he decided to help me by bringing me. Aside from that, I have not received any form of help from anybody again." -Seamstress, 20-29 years.

To corroborate this, another respondent said: "Please no, there is no one, even up till now I don't have anybody who will pay my bills for me." -Hairdresser, 20-29 years.

The findings show a larger proportion of the post-abortion care clients received support from their family and friends as opposed to their counterparts who did not receive any support. This could be ascribed to the family support system in many sub-Saharan African countries where families turn to be each other's keepers.

\section{Discussion}

The study sought to explore the pregnancy termination experiences of women seeking post-abortion care services. The results showed that women who induced their abortions relied on medications to terminate their pregnancies. This could be as a result of the fact that medical abortion offers women the privacy to terminate their pregnancies without having to face several people in a health facilities. This also helps reduce stigma; as when done successfully, no one might even know of it. This is in line with a study conducted by Sedgh [17], which revealed that most women who sought abortion induced abortion on their own. Other studies also observed that women generally preferred medical abortions to other pregnancy termination techniques $[20,19]$. Given the high usage of medical abortion at home, it is important to increase education and awareness for women and pharmacists on the right medication for medical abortion to reduce the risk of women using illegal methods of abortions.

The study also found that the spontaneous termination of pregnancies among women was mostly as a result of engaging in strenuous physical activities. This is, however, not medically proven as it is from the respondents' layman point of view. This corroborates the findings of Wong et al., [29] which indicated that there is an elevated risk for women working in jobs that required the use of strenuous physical energy. It would, therefore, be imperative for women to know their health conditions before indulging in certain activities while pregnant.

It was observed that whereas some factors influenced the time a woman decided to seek professional health care for post-abortion complications, the most influential factor was the severity of the pain being felt from the complication. Stigma and poverty, nonetheless, also played a vital role in influencing the decision-making process. The delay by women seeking post-abortion care services could be attributed to the fact that women would like to suffer in silence to protect their privacy rather than suffer the stigma attached to abortion since in most developing countries, abortion is a religious, moral and political issue 
instead of a medical or public health issue [3]. This corroborates the findings of Finer and Henshaw [30] who argued that most women allow a longer time to elapse between abortion and the time for seeking care. This could be attributed to the stigma associated with induced abortion in developing countries coupled with the laws that render an abortion legal only under certain conditions [7].

Despite the important findings made from this study, it is imperative to indicate its possible limitations. For instance, the study was delimited to clients from only one health facility, which limited the study from being generalized to the general Ghanaian society. This, however, did not limit the validity and trustworthiness of the study since the necessary steps to make the instrument valid and trustworthy were followed.

\section{Conclusion}

The study established that women who induced their abortions preferred to do so through medical abortion. However, spontaneous abortion was usually as a result of pregnant women carrying out activities that required the use of excessive energy and women traveling on bumpy roads. The study also concluded that stigma and financial challenges hindered women from seeking PAC services. Furthermore, the paper has shown that women who suffer complications from pregnancy terminations and seek PAC services usually receive financial and other forms of support from their families and partners.

To encourage women to seek early treatment, the Ministry of Health and the Ghana Health Service should take pragmatic steps in educating women on the dangers associated with delayed post-abortion care services and the factors that expose women to spontaneous abortions.

\section{Abbreviations}

PAC - Post-Abortion Care

D\&C - Dilation and Curettage

D\&E - Dilation and Evacuation

GSS - Ghana Statistical Services

\section{Declarations}

\section{Ethics approval and consent to participate}

Ethical clearance for the study was obtained from the Ghana Health Service Ethical Review Committee (GHS-ERC: 14/10/15). Permission to conduct the study was also obtained from the management of the hospital. Clients who agreed to take part in the study were required to sign a consent form before the interview was conducted. 


\section{Consent to publication}

Not applicable

\section{Availability of Data and Materials}

All data generated or analyzed during this study are included in this published article

\section{Competing Interests}

The authors declare that they have no conflicts of interest.

\section{Funding}

No funding received

\section{Authors' Contributions}

KSA conceived the study. KSA, EKMD, and AKK designed and performed the analysis. KSA drafted the manuscript. EKMD and AKK reviewed the manuscript and provided substantial contributions to the interpretation of data and discussion of findings which greatly improved the intellectual content. All authors read the final manuscript and approved it.

\section{Acknowledgments}

We acknowledge the immense assistance from the Volta Regional Hospital, Ho and the post-abortion care clients who participated in this research.

\section{Disclosure}

The paper does not represent the views of the Volta Regional Hospital.

\section{Author information}

Kenneth Setorwu Adde ${ }^{1}$ (kenneth.adde@stu.ucc.edu.gh)

Eugene Kofuor Marfo Darteh ${ }^{1}$ (edarteh@ucc.edu.gh)

Akwesi Kumi-Kyereme ${ }^{1}$ (akkyereme@ucc.edu.gh)

${ }^{1}$ University of Cape Coast

${ }^{1}$ Department of Population and Health, College of Humanities and Legal Studies, University of Cape Coast, Cape Coast, Ghana

\section{References}


[1] Kim, C. R., Tuncalp, O., Ganatra, B., \& Gulmezoglu, A. M. WHO Multi-Country Survey on Abortion-related Morbidity and Mortality in Health Facilities: study protocol. BMJ Global Health,2016; 1(3), e000113.

[2] Rominski, S. D., Bell, A. J., Yawson, A. E., Nakua, E. K., \& Moyer, C. A. Is abortion justified to save the life or health of a woman? Evidence of public opinion from Accra, Ghana. International Journal of Gynecology and Obstetrics, 2019; 147, 115-119. doi:DOI: 10.1002/ijgo.12927

[3] Sedgh, G., Bearak, J., Singh, S., et al.. Abortion incidence between 1990 and 2014: global, regional, and subregional levels and trends. The Lancet,2016;388(10041), 258-267.

[4] Aniteye, P., \& Mayhew, S. Attitudes and experiences of women admitted to hospital with abortion complications in Ghana. African Journal of Reproductive Health, 2011; 15(1), 47-55.

[5] Ghana Health Services. (2006). Reproductive and child health annual programme of work. Ghana Health Services, Reproductive and Child Health.

[6] Oduro G.Y, \&Otsin M.N.A. "Abortion - it is my own body": Women's narratives about influences on their abortion decisions in Ghana. Health CareWomen Int 2014;35:7-9.

[7] Morhee, R., \& Morhee, E. Overview of the law and availability of abortion services in Ghana. Ghana Medical Journal, 2006; 40(3), 80-86.

[8] Gaye, A., Diop, A., Shochet, T., \& Winikoff, B. Decentralizing post-abortion care in Senegal with misoprostol for incomplete abortion. International. Journal of Gynaecology and Obstetrics, 2014; 126(3), 223-226.

[9] Dickens, B. M. Post-abortion care:Ethical and legal duties. Ethical and legal issues in reproductive health. 2019; doi:DOI: 10.1002/ijgo.12951

[10] Warriner, I. K., \& Shah, I. H. Preventing Unsafe Abortion and its Consequences: Priorities forResearch and Action, New York: Guttmacher Institute. 2006.

[11] Finer, L. B., Frohwirth, L. F., Dauphinee, L. A., Singh, S., \& Moore, A. M. Reasons U.S. women have abortions: Quantitative and qualitative perspectives. Perspectives on Sexual and Reproductive Health,2005; 37, 110-118.

[12] Mpangile, G. S., Leshabari, M. T., \& Kihwele, D. J. Induced abortion in Dares Salaam, Tanzania: the plight of adolescents. In: Mundigo, A. I. and Indriso, C. (eds). Abortion in the developing world. New Delhi: Vistaar for the World Health Organization, 1999; 387-403.

[13] WHO. Unsafe abortion, Global and Regional Estimates of the Incidence of Unsafe Abortion and Associated Mortality in 2003. (5th ed). Geneva: WHO. Retrieved on February $6^{\text {th }}$ June 2016 from http://www.who.int/reproductivehealth/publications /unsafeabortion_2008/ua_estimates03.pdf. 2007 
[14] Ghana Statistical Services (GSS), Ghana Health Service (GHS), \& ICF International. Ghana maternal health survey 2017. Calverton, Maryland: GSS, GHS, and Macro International. 2017.

[15] Ahiadeke, C. Induced abortion in the context of reproductive change in Ghana. In Agyei-Mensah, S., Casterline, J. B. \& Agyeman, D. K. (Eds). Reproductive Change in Ghana: Recent Patterns and Future Prospects, University of Ghana, Legon, 2005; 178-192.

[16] Geelhoed D. W., Visser L. E., Asare, K., Schagen van Leeuwen, J. H., \& van Roosmalen J. Trends in maternal mortality: A 13-year hospital-based study in rural Ghana. Eur J Obstet Gynecol Reprod Biol; 2003; 107, 135- 139.

[17] Sedgh, G. Abortion in Ghana, In Brief, New York: Guttmacher Institute, 2010; No. 2.

[18] Ahiadeke, C. Incidence of induced abortion in southern Ghana. International Family Planning Perspectives, 2001; 96-108.

[19] Blum, J., Diop, A., Frimpong, P., \& Taylor, J. Medical Abortion in Ghana: Feasibility and Acceptability of Mifepristone and Misoprostol in low resourced areas'. Unpublished study conducted in La General Hospital and Koforidua Government Hospital, 2007. 2005.

[20] Ghana Statistical Services (GSS), Ghana Health Service (GHS), \& ICF International. Ghana maternal health survey 2007. Calverton, Maryland: GSS, GHS, and Macro International. 2009.

[21] Ghana Health Service. National Reproductive Health Policy: Standards and Protocols.2013.

[22] Ghana Health Service. Prevention and management of unsafe abortion: Comprehensive abortion care services. Standards and protocols. Accra: GHS; 2006.

[23] Hessini, L., Brookman-Amissah, E., \& Crane, B. B. Global policy change and women's access to safe abortion: the impact of the World Health Organization's guide in Africa. African Journal of Reproductive Health, 2006; 10(3), 14-27.

[24] Morhe, E. S., Tagbor, H. K., Ankobea, F. K., \&Danso, K. A. Reproductive experiences of teenagers in the Ejisu-Juabeng district of Ghana. International Journal of Gynecology \& Obstetrics, 2012; 118(2), 137-140.

[25] Sundaram, A., Juarez, F., Bankole, A., \& Singh, S. Factors Associated with Abortion-Seeking and Obtaining a Safe Abortion in Ghana. Studies in Family Planning, 2012;43(4), 273-286.

[26] Esia-Donkoh, K., Darteh, E. K., Blemano, H., \&Asare, H. Who cares? Pre and post-abortion experiences among young females in Cape Coast Metropolis, Ghana. African journal of reproductive health, 2015; 19(2), 43-51.

[27] Rominski, Sarah D., Emmanuel SK Morhe, and Jody Lori. "Post-abortion contraception choices of women in Ghana: A one-year review." Global public health, 2015; 10(3), 345-353. 
[28]Guest G, Bunce A, Johnson L: How many interviews are enough? An experiment with data saturation and variability. Field Methods. 2006, 18 (1): 59-82. 10.1177/1525822X05279903.

[29] Wong, E. Y., Ray, R., Gao, D. L., et al., Physical activity, physical exertion, and miscarriage risk in women textile workers in Shanghai, China. American journal of industrial medicine, 2010; 53(5), 497-505.

[30] Finer, L. B., \& Henshaw, S. K. Disparities in rates of unintended pregnancy in the United States, 1994 and 2001. Perspectives on sexual and reproductive health, 2006; 38(2), 90-96.

\section{Supplementary Files}

This is a list of supplementary files associated with this preprint. Click to download.

- interviewguide.pdf

- interviewguide.pdf

- interviewguide.pdf 